

\section{REVISTA \\ TEORÍA Y PRÁCTICA \\ DE LA \\ ARQUEOLOGÍA HISTÓRICA LATINOAMERICANA}

ISSN: 2250-866X (impreso) | ISSN: 2591-2801 (en línea)

AÑo VIII, VOLUMEN 8, PRIMAVERA DE 2019

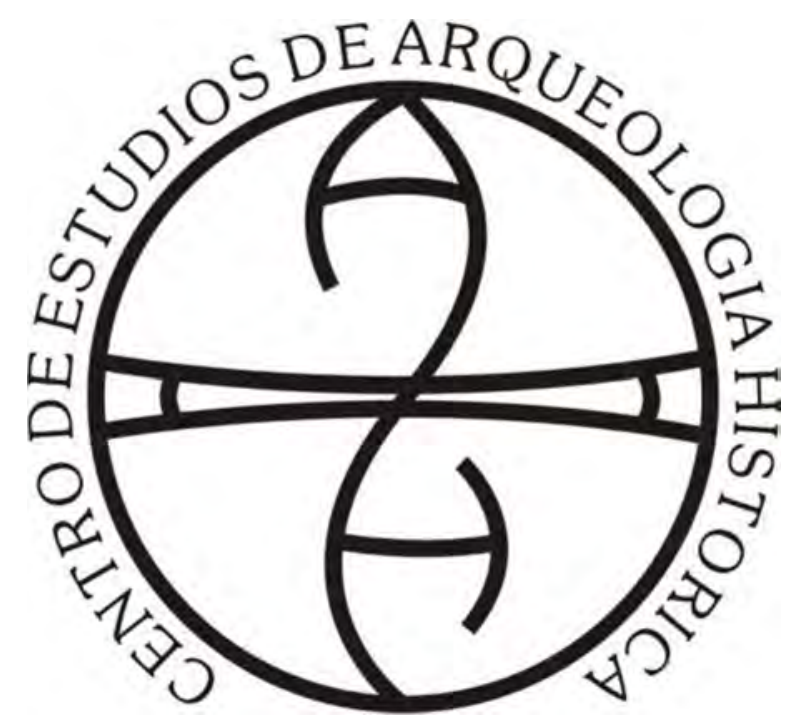

Centro de Estudios de Arqueología Histórica

FACULTAD DE HUMANIDADES Y ARTES | UNIVERSIDAD NACIONAL DE ROSARIO 
(Universidad Nacional de Rosario, Universidad Nacional de Río Cuarto,

Universidad Nacional de San Juan, Universidad de la República, Universidad Nacional de Trujillo)

\author{
AUTORIDADES DE LA UNIVERSIDAD NACIONAL DE ROSARIO \\ RECTOR: Lic. Franco Bartolacci \\ VICE-RECTOR: Od. Darío Macía \\ SECRETARIO GENERAL: Prof. José Goity \\ SECRETARIA ACADÉMICO Y DE APRENDIZAJE: Dr. Marcelo Vedrovnik \\ SECRETARÍA DE CIENCIA TECNOLOGÍA E INNOVACIÓN \\ PARA EL DESARROLLO: Ing. Guillermo Montero.
}

\author{
AUTORIDADES DE LA FACULTAD DE HUMANIDADES Y ARTES \\ DECANO: Prof. Alejandro Vila \\ VICEDECANA: Prof. Marta Varela \\ SECRETARIA ACADÉMICA: Dra. Marcela Coria
}

\author{
AUTORIDADES DEL CENTRO DE ARQUEOLOGÍA HISTÓRICA \\ DIRECTORA: Dra. Ana Rocchietti \\ SECRETARIA: Prof. Nélida de Grandis \\ PROSECRETARIA: Lic. Marianela Bizcaldi
}

DIRECTORAS - EDITORAS:

Dra. Ana Rocchietti y Prof. Nélida De Grandis

SECRETARIA DE EDICIÓN: Dra. Irene Dosztal

Este número es co-edición de las ponencias

del VIII Congreso Nacional de Arqueología Histórica (2018) entre:

Centro de Estudios en Arqueología Histórica: Directora Ana Rocchietti

Centro de Estudios en Arqueología Regional: Director Fernando Oliva

Centro de Estudios en Arqueología Subacuática: Directora Mónica Valentini

Departamento de Arqueología, Escuela de Antropología: Director Fernando Oliva
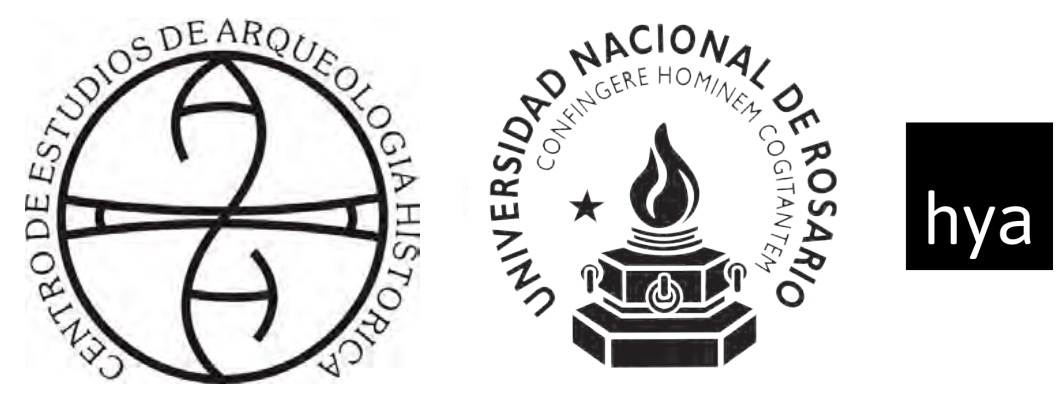

Facultad de Humanidades

y Artes_UNR

Comité Permanente de los Congresos Nacionales de Arqueología Histórica

Dr. Daniel Schávelzon (Universidad Nacional de

Buenos Aires)

Prof. María Teresa Carrara (Universidad Nacional de Rosario)

Prof. Carlos Baldassarre (Museo Municipal de Río

Grande, Tierra del Fuego) in memoriam

Dr. Mariano Ramos (Universidad Nacional de Luján, CONICET)

Dr. Horacio Chiavazza (Universidad Nacional de Cuyo)

Dra. Ana María Rocchietti (Universidad Nacional de

Rosario, Universidad Nacional de Río Cuarto)

Lic. Facundo Gómez Romero (Universidad Autónoma

de Barcelona)

\section{Comité Científico}

Dra. Tânia Andrade Lima (Universidade Federal do Rio de Janeiro)

Prof. Réginald Auger (CELAT/Département des

Sciences Historiques, Université Laval, Canadá)

Dr. Roberto Bárcena (Universidad Nacional de Cuyo, CONICET)

Dra. Marta Bonaudo (Universidad Nacional de Rosario, CONICET)

Dr. Leonel Cabrera (Universidad de la República, Uruguay)

Dr. Luis María Calvo (Universidad Católica de Santa

$\mathrm{Fe})$

Prof. Juan Castañeda Murga (Universidad Nacional de

Trujillo, Perú)

Dr. Carlos Ceruti (Museo de Ciencias Naturales y

Antropología "Prof. Antonio Serrano". Paraná)

Dr. Horacio Chiavazza (Universidad Nacional de Cuyo)

Dra. Silvia Cornero (Universidad Nacional de Rosario)

Prof. Pedro Paulo Funari (Universidade Estadual de

Campinas, Brasil)

Lic. Jorge A. Gamboa Velásquez (Universidad Nacional

Santiago Antuñez de Mayolo, Perú)

Dr. Eduardo Alejandro García (Universidad Nacional de

San Juan, CONICET)

Prof. Nélida De Grandis (Universidad Nacional de

Rosario)

Dr. Juan Bautista Leoni (Universidad Nacional de

Rosario, CONICET)

Dra. Amancay Martínez (Universidad Nacional de San

Luis)

Dra. Catalina Teresa Michieli (Universidad Nacional de

San Juan, CONICET)

Lic. Fernando Oliva (Universidad Nacional de Rosario)

Ing. Adrián Pifferetti (Universidad Tecnológica
Nacional Regional Rosario)

Dr. Mariano Ramos (Universidad Nacional de Luján, CONICET)

Dra. Ana María Rocchietti (Universidad Nacional de Rosario)

Dr. Daniel Schávelzon, (Universidad Nacional de Buenos

Aires, CONICET)

Dra. Carlota Sempé (Universidad Nacional de La Plata)

Dr. Mario Silveira (Universidad Nacional de Buenos Aires)

Dra. Silvia Simonassi (Universidad Nacional de Rosario)

Dra. Alicia Tapia (Universidad Nacional de Buenos Aires, Universidad Nacional de Luján)

Lic. Mónica P. Valentini (Universidad Nacional de Rosario)

Agrim. Benito Vicioso (Universidad Nacional de Rosario)

\section{Evaluaron este volumen}

Roberto Bárcena, María Teresa Boschin, Leonel Cabrera, Ulises Camino, María Rosa Carbonari, Carlos Ceruti, Horacio Chiavazza, Nicolás Ciarlo, Silvia Cornero, Eduardo Crivelli, Javier García Cano, Martín Gentinetta, María Laura Gili, Carlos Landa, Matilde Lanza, Melina Malandrino, Sebastián Pastor, Victoria Pedrotta, Josefina Piana,

Mercedes Podestá, Mariano Ramos, Daniel Schavelzon, Diana Tamburini, Mónica Therrien, Mónica Valentini y María Teresa Boschin

\section{Diseño y diagramación}

Eugenia Reboiro

(eugenia.reboiro@gmail.com)

\section{Curadoría}

Ana Rocchietti e Irene Dosztal

Foto de tapa: imagen del texto de Croce.

\section{Propietario responsable:}

Facultad de Humanidades y Artes, Universidad Nacional de Rosario Centro de Estudios de Arqueología Histórica

Entre Ríos 758. Rosario, provincia de Santa

Fe (2000). Argentina.

Telf.: +54 (0341) 4802670

E-mail: ceahunr@gmail.com

Decreto Ley 6422/57 de Publicaciones

Periódicas 


\section{Índice}

Editorial.

Arqueología histórica: programa de investigación y dimensiones epistemológicas...9 Ana María Rocchietti

De lozas, iglesias y machaqueños. Primeros pasos hacia una arqueología histórica en la cuenca norte del río desaguadero (La Paz, Bolivia).

Salvador Arano Romero

Identificación arqueológica de acciones militares en el campo de batalla de Cepeda, 1859.

Juan B. Leoni, Lucas H. Martínez, Cecilia Arias Morales, Daniela Cadenas,

Faustino Godoy, Mauro Ganem, María de la Paz Blanche y Héctor Meletta

Para una historia de la arqueología urbana en Buenos Aires (1848-1910) .59

Daniel Schávelzon y Francisco Girelli

Definiendo la cultura material colonial a partir de colecciones arqueológicas del Museo de La Plata.

Melina Bednarz, Julieta Penesis y Ana Igareta

La tangibilidad del conflicto: Arqueología del presente en las fronteras del norte santafesino....

Paula Del Río y Silvia Cornero

Arqueología histórica: evidencia material en el paisaje del establecimiento jesuita "La saladilla” (quebrada de Saladillo, La Rioja, Argentina). Juan Carlos Giuliano, Maira Ayelén Carrizo y María Soledad Gheggi

Graffiti ¿evidencia arqueológica o acto vandálico?. Camila Oliva

Arqueología histórica de los sistemas hidráulicos de la Hacienda

Jalpa de Cánovas, Guanajuato, México, y su relación con el sistema-mundo, Siglos XVIII-XIX

José Alberto Aguirre Anaya y Edgar Quispe Pastrana 
Paseo del bajo: una trinchera en la historia de Buenos Aires.................................143 Federico Martín Croce

Diferentes concepciones del patrimonio y su aplicación a la Casa Histórica de Tucumán también llamada Casa de la Independencia........................................155 Guillermo Etchevers

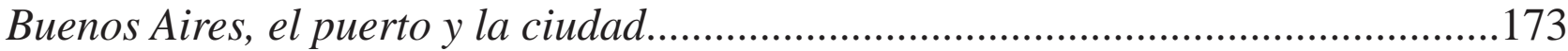
Mónica P. Valentini y Javier García Cano 


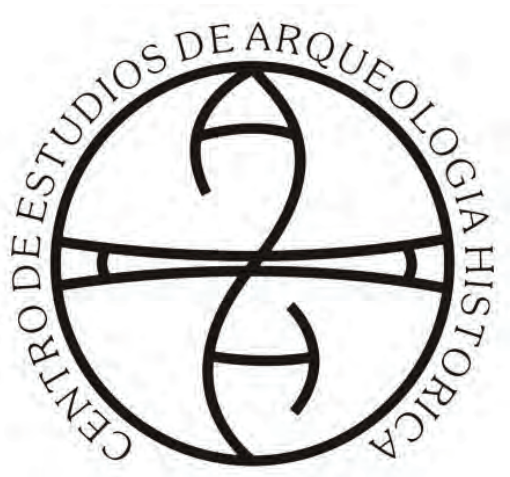

Centro de Estudios de Arqueología Histórica

Universidad Nacional de Rosario
Teoría y Práctica de la Arqueología Histórica

Latinoamericana | Año VIII. Vol. 8 | 2019

Revista del Centro de Estudios de Arqueología

Histórica, Facultad de Humanidades y Artes,

Universidad Nacional de Rosario

https://teoriaypracticaah.unr.edu.ar/index.php/index

ceahunr@gmail.com

ISSN en línea: 2591-2801

ISSN versión impresa: 2250-866X

\title{
DIFERENTES CONCEPCIONES DEL PATRIMONIO Y SU APLICACIÓN A LA CASA HISTÓRICA DE TUCUMÁN TAMBIÉN LLAMADA CASA DE LA INDEPENDENCIA
}

\author{
Guillermo Etchevers*
}

\begin{abstract}
Resumen
En relación las diferentes posturas que actualmente coexisten en el mundo de las gestiones patrimoniales, resulta interesante analizar el caso de la Casa Histórica de Tucumán, dada su condición de símbolo y referente de la independencia nacional, pero también como bien patrimonial a pesar de las transformaciones que la alejan tanto de su estado original.

Se trata de un ejemplo único en relación a la importancia y trascendencia que fue cobrando durante el tiempo, ya que fue sede de la declaración de la independencia; pero habiendo sido abandonada e ignorada en sus primeras épocas, fue sujeto de un proceso fluctuante en los criterios sobre su valoración, que la mantuvieron sujeta a decisiones contrapuestas y en ocasiones contradictorias que derivaron en paulatinas transformaciones, y posteriores abandonos, hasta alcanzar graves estados de ruina y su casi total demolición. En la década de 1940, a raíz de políticas estatales que buscaban símbolos de identificación nacional, se buscó recuperar el bien mediante una reconstrucción total, procurando respetar los criterios de intervenciones imperantes en el momento. Intervenciones que despertaron la polémica y oscilaron entre su más cruda interpretación como un "falso histórico", hasta su plena justificación como símbolo necesario de identidad nacional, superando la validez de originalidad del propio objeto.

La casa de Tucumán, constituye un claro ejemplo que permite el desarrollo de las transformaciones que la fueron afectando, así como la reflexión sobre que acciones pudieron ser las más idóneas; y considerando que el edificio actual constituye una reconstrucción del original, la posibilidad de que haya adquirido valor propio, no por el símbolo que constituye, sino por el propio edificio. Esta reconstrucción, es un referente de la tendencia neocolonial emergente hacia la década de 1940 y del prestigio de los profesionales que intervinieron. Y constituye un nuevo bien patrimonial por la propia antigüedad que conlleva, y por lo tanto susceptible -también la reconstrucción- de ser intervenida patrimonialmente.
\end{abstract}

* Jefe de trabajos prácticos en Historia de la Arquitectura Cátedra PERNAUT - Facultad de Arquitectura Diseño y Urbanismo de la Universidad de Buenos Aires. Representante técnico Dirección Nacional de Diseño y Planificación de Obras. guillermo. etchevers@gmail.com 
De este modo, no solo intentamos analizar las posturas patrimoniales sobre su situación actual, sino también sobre las acciones previas y su contexto histórico, lo cual hace necesaria una breve reseña histórica, que informe cronológicamente la accidentada y cambiante historia de sus intervenciones, o en que sectores de la casa se fueron dando. Y para ello, también surge la necesidad de una breve descripción de la casa, que identifique los sectores y las áreas que fueron sujeto de dichas acciones, así como su materialidad y diferentes calidades constructivas.

Palabras clave: “Casa de Tucumán” - Argentina - Posturas patrimoniales - Historia arquitectónica

\begin{abstract}
In relation to the different positions that currently coexist in the world of heritage management, it is interesting to analyze the case of the Historic House of Tucumán, given its status as a symbol and referent of national independence, but also as a patrimonial asset despite the transformations that distance it from its original state.

It is a unique example in relation to the importance and transcendence that it was gaining during the time, since it was the seat of the declaration of independence; but having been abandoned and ignored in its early epochs, was subject to a fluctuating process in the criteria for its assessment, which kept it subject to conflicting and sometimes contradictory decisions that led to gradual transformations, and subsequent abandonments, until they reach serious stages of ruin and their almost total demolition. In the 1940s, as a result of State policies that sought symbols of national identification, efforts were made to recover the good through a total reconstruction, trying to respect the criteria of interventions prevailing at the time. Interventions that aroused the controversy and ranged from their crudest interpretation as a historical fake, to their full justification as a necessary symbol of national identity, overcoming the validity of originality of the object itself. The house of Tucumán is a clear example that allows the development of the transformations that were affecting it, as well as the reflection on what actions could be the most suitable; and considering that the current building constitutes a reconstruction of the original, the possibility that it has acquired its own value, not by the symbol it constitutes, but by the building itself. This reconstruction is a reference of the emerging neo-colonial trend towards the 1940s and the prestige of the professionals who intervened. And it constitutes a new patrimonial asset because of the antiquity that it entails, and therefore susceptible-also the reconstruction-to be intervened patrimonially.

In this way, we try not only to analyze the patrimonial positions on their current situation, but also on the previous actions and their historical context, which makes necessary a brief historical review, to report chronologically the rugged and changing history of his interventions, or in which sectors of the house they were given. And for this, there is also a need for a brief description of the house, which identifies the sectors and areas that were the subject of these actions, as well as their materiality and different constructive qualities
\end{abstract}

Keywords: “Tucumán House” - Argentina - Heritage positions - Architectural history

\title{
Breve descripción de la casa
}

La casa responde al sistema tradicional de dos patios alineados a lo largo de un eje y separados por un pabellón. Siendo el de adelante el principal, estaba rodeado por las habitaciones de la familia, la sala y el comedor; y el posterior cumplía las funciones de servicio, con la huerta, el pozo de agua y las galerías para las habitaciones de servidumbre (figura 1). 


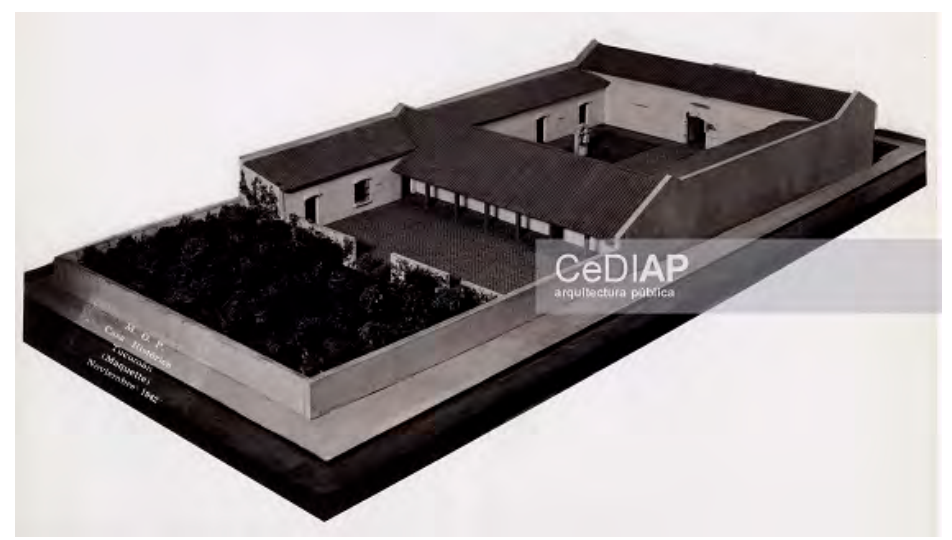

Figura 1. Modelo digital de la versión original (Fuente: CeDIAP ${ }^{1}$

"Respecto a los materiales constructivos, se puede observar una gran homogeneidad en toda la casa, con cubiertas de tejas sobre cañizo y muros de tapial revocados con barro y blanqueados a la cal, ${ }^{2}$ excepto en el sector correspondiente al zaguán y los cuartos que lo flanqueaban, cubiertos con bóvedas de ladrillo apoyadas sobre muros probablemente también de ladrillos” (Marinsalda, 1999:161), como se ve en la foto de Paganelli (ver figura. 5).

Independientemente de las bóvedas o el cañizo, y de la tapia o los ladrillos, este tipo constructivo requería de un continuo trabajo de mantenimiento, para que las intensas lluvias no provocaran su deterioro, lo que explica la decadencia de la casa con el correr de los años. (fig.2)



Figura 2: Primer patio- Foto de Paganelli. (Fuente: CeDIAP)

Lo único en la casa con decoración, es el icónico portal barroco con sus conocidas columnas salomónicas, el zaguán y a las porterías que lo flanquean (fig. 3). Se trataba de una gran puerta de 2 hojas con un arco escarzano, rodeada por dos conjuntos de pilastras y medias columnas salomónicas a cada lado que, al superar la altura de la cornisa general, conforman una ambigüedad entre capitel y el residuo de lo que sería un gran entablamento, cuya cornisa continúa entre ambos conjuntos, definiendo un espacio entre ella y la puerta, equiparable a un frontis con su tímpano. 




Figura 3. La Casa Histórica de Tucumán en su estado actual (Fuente: Imagen del autor).

Dicha cornisa superior, es mixtilínea, y es la que le da la identidad icónica al conjunto del portal, ya que originalmente ese tímpano albergaba algún un tipo escudo del cual no se tienen certezas, ya que solo se ve en la foto de Paganelli (ver figura 5). Donde ese sector se ve apenas revocado o con el ladrillo visto.

Actualmente alberga el escudo nacional que coincide perfectamente con la curvatura original, como si ella hubiese sido diseñada para este escudo y no el primitivo. El portal se ampliaba a ambos lados con las dos ventanas laterales de menor complejidad y un ático que acompañaba la altura del orden mayor del sector central.

Encuadrado por el gran portal y a través zaguán de entrada, se vislumbran algunas zonas del primer patio, que mantienen vigente una de las invariantes de la arquitectura hispanoamericana; la relación interior- exterior, esa constante dependencia de los espacios exteriores o patios, y esa situación que nos lleva desde el exterior (previo zaguán) nuevamente al exterior (figura 3).



Figura 4. El patio en su estado actual (Fuente: CeDIAP) 


\section{Reseña histórica de la casa}

La accidentada y cambiante historia del edificio podría entenderse básicamente en seis etapas donde también tubo usos diferentes, el original de vivienda típica del siglo XVIII y usado para tal fin, la del edificio casi sin alteraciones pero alquilado por el estado nacional, la del edificio devuelto a sus herederos quienes la dejan en estado ruinoso y con las habitaciones del 2do patio reconstruidas, la de la compra por el estado nacional y cambio del sector más icónico por una fachada neoclásica (ver figura 6), la del S XX con el pabellón en estilo neo renacentista (ver figura 8), y finalmente la de la nueva reconstrucción imitando la original como objeto patrimonial (ver figura 3).

A estas etapas se las podría ordenar cronológicamente de la siguiente manera:

1- Construida en la década de 1760, perteneció a una familia numerosa que, no solo le dio uso de vivienda sino también como talleres de producción.

2- Después de la batalla de Tucumán (1812) la casa se alquiló para funciones del estado revolucionario, y luego de 1817 retorno a su función de vivienda y locales de alquiler. Durante esta etapa, si bien se espera que dado su intenso uso hubiese sufrido alteraciones, solo se demolió un muro en el pabellón central, para así agrandar la sala en la que sesionara el Congreso. Entre las tareas de mantenimiento y reparaciones, se cambiaron los cañizos por tablones y se repintaron las carpinterías (por cambio de azul a rojo).

3- El regreso al uso de vivienda duró hasta 1875, periodo en se demolieron algunos locales del segundo patio, que estaban en estado ruinoso. Su precaria calidad -con tapia y casi sin cimientos-, explica su endeble estabilidad y su reemplazo por otra edificación.

Excepto esas intervenciones menores, la situación económica de los herederos no permitió grandes mantenimientos, y ya cercana a sus 100 años -considerando su tipo constructivo- la casa alcanzaba una fase terminal de su vida útil. (Figura 5).

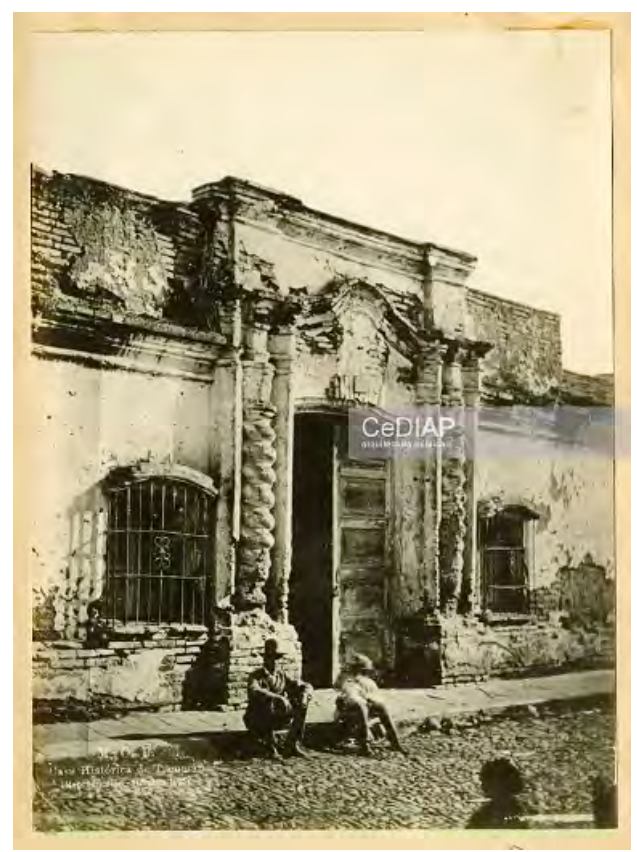

Figura 5. El portal original-Foto de Paganelli. (Fuente: CeDIAP) 
4- En 1869 fue sancionada la ley autorizando al Poder Ejecutivo Nacional a adquirir la casa y hacerse cargo de su conservación, que en 1875 implico el proyecto de transformar la casa en Oficina de Correo, Telégrafos y Juzgado Federal, para lo cual se demolieron y reconstruyeron gran parte de los cuartos y galerías del primer patio, como así también la totalidad de la fachada eliminando el portal colonial, sustituyéndolo por una fachada neoclásica, acorde con el criterio oficial de la época. (Figura 6).

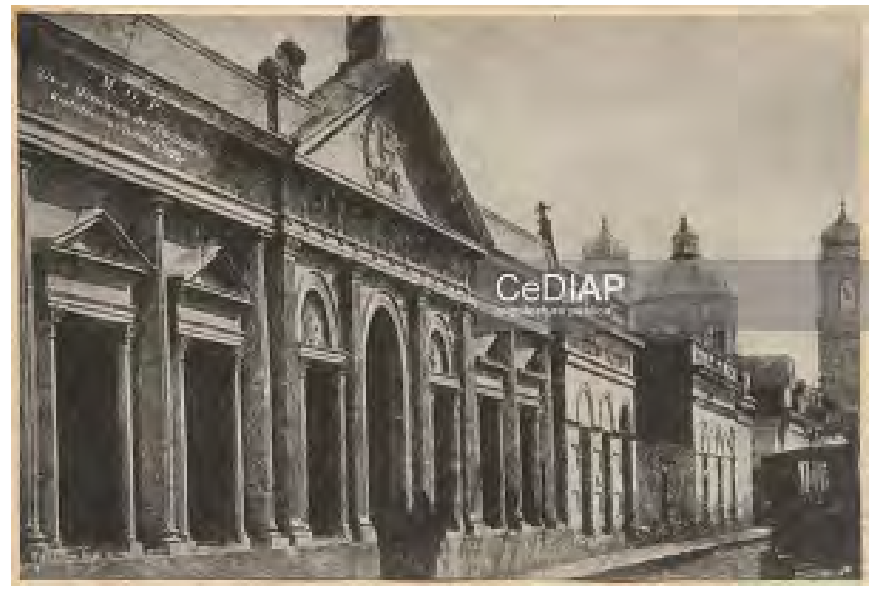

Figura 6. La fachada Neoclásica. (Fuente: CeDIAP)

“En el corto periodo transcurrido entre los años 1869 y 1876, junto con la destrucción del portal colonial y el frente, se van a producir los hechos y elementos fundamentales que permitirán construir la historia mítica de la casa, en un proceso que encontrara su punto de inflexión en 1916 y que levara a la materialización de este mito en 1943” (Marinsalda, 1999:162).

5- A pesar de las demoliciones y reconstrucciones de 1875, hacia 1904 el edificio estaba de nuevo en estado ruinoso y en esta ocasión se demolió casi todo. Es decir, no se trató de una modificación sino de una obra totalmente nueva para alojar lo único que se conservó, el salón histórico en el cuerpo central (Figura 7).

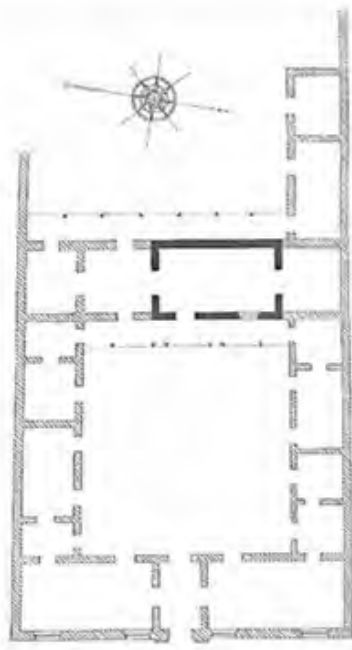

Figura. 7. Único salón sin demoler en 1904. (Fuente: Anales 31-31 del IAA³ 
Esta obra se trató de un pabellón ecléctico de carácter italianizante, pero con grandes aventanamientos y techo de vidrio que, al ubicarse en torno al mencionado salón, dejaba vacío el espacio que originalmente ocupaba el cuerpo frontal de la casa. De esta forma, el pabellón conto con un patio de ingreso, adornado con jardines y esculturas, que conformaron una gran antesala para su fachada monumental y su pórtico. (Figura 8)



Figura 8. El pabellón ecléctico. (Fuente: CeDIAP)

6- En 1941 la Casa de la Independencia fue declarada Monumento Histórico Nacional, su desaparición material era casi absoluta, se inició la reconstrucción de la Casa del Congreso de 1816 bajo proyecto de Buschiazzo. El inconveniente es que, para su reconstrucción, debían valerse solo de las 2 fotografías de Paganelli (ver fig. 4, 5), y una tercera anónima, (fig.9) y los relevamientos previos a la fachada neoclásica, que fueron cercanos a 1870, por lo que se asumió que las partes más representativas de la casa no habían sufrido cambios entre 1816 y 1870 y se realizaron los trabajos que reconstrucción según la poca documentación recolectada, omitiendo lo que no había sido registrado, como la galería norte del primer patio o dejar sin pintar las carpinterías por desconocimiento del color. 


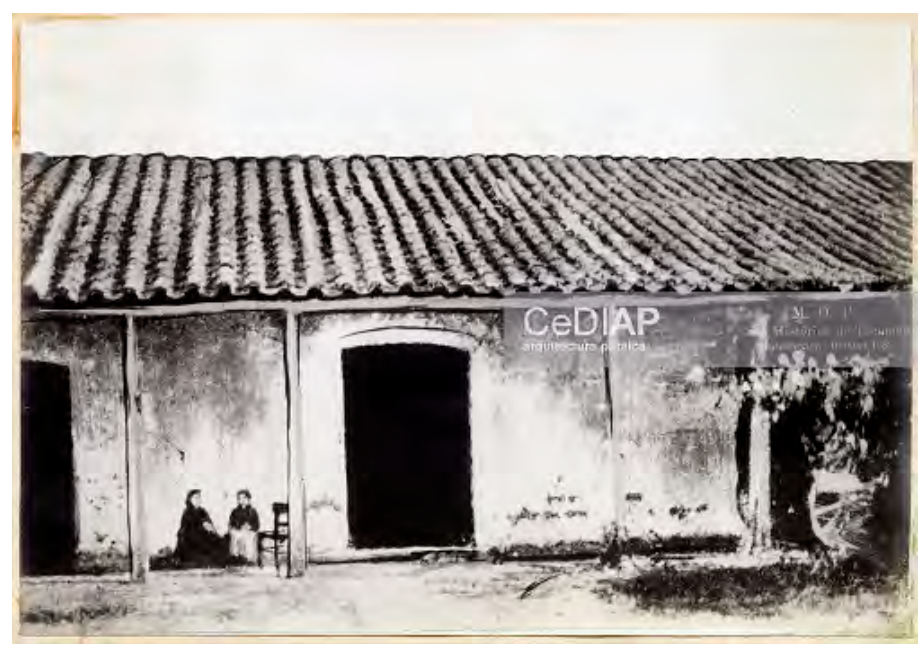

Figura 9. Primer Patio- Fotografía anónima. (Fuente: CeDIAP)

Su reconstrucción se completó en 1943, pero en 1966 y 1976 se complementó su condición de museo con la demolición de la casa posterior e incorporación de sus terrenos y luego lo mismo con los terrenos a ambos lados como espacio de amortiguación y de exposición de las obras de las etapas intermedias.

\section{Entre el monumento y el símbolo}

Esta breve reseña histórica, tuvo la intención de detallar los procesos que vivió la casa, desde sus orígenes hasta su reconstrucción, intentando no emitir juicios de valor sobre cada acontecimiento, sino ofrecer el material que nos permita ahora sí, elaborar una reflexión sobre cómo pudieron haber sido sus procesos, si fueron evitables o no, o al menos convenientes. La intención es hacer un contrapunto entre la perspectiva de nuestros días y los criterios que se manejaban en cada una de las épocas que la afectaron; en donde la casa paso de ser un edificio de utilidad cotidiana, hasta su transformación en un símbolo que supera la necesidad del objeto físico.

De este modo, el caserón de dos patios y una huerta posterior, ya contaba con antigüedad cuando fue sede de la declaración de la independencia (dese 1760), y estaba ejecutado con materiales precarios y efímeros (tapia, adobe, revoques de barro, techumbres de tejas sobre cañizo, etc.), así como sufrió cambios de materiales. También la demolición del pabellón del fondo y el reemplazo del sector de ingreso por una fachada neoclásica, y finalmente la demolición completa que preservo solo el salón de la proclamación.

Es fácil entender que era un tipo de construcción que no mucho tiempo podría resistir; al menos con un clima húmedo como el de Tucumán con largas temporadas de lluvias y, dependiendo de un mantenimiento casi equivalente a una reconstrucción crónica. Pero también es cierto que otras regiones de América conservan aun edificaciones en tapia y hasta en quincha, que son inclusive más antiguas, aunque se ubican en climas extremadamente secos que no degradan tan fácilmente sus muros tan solubles; y que también se sostienen por un mantenimiento (o reconstrucción) crónica con los que no conto nuestro ejemplo de estudio.

También observamos que dentro del conjunto hubo diferentes categorías, y como tales, diferentes calidades constructivas, con algunos sectores muy precarios que inclusive resistieron menos que el resto del conjunto. 
Dicho en otras palabras... es natural la reacción de rechazo, al leer que en 1875 fue demolido del icónico portal de la entrada y reemplazado por una fachada neoclásica, (ver figura 6) y más aún cuando luego, con la obra de 1905, (conservando solo el salón) se completó la demolición de casi todo el conjunto (ver figura 7). Pero adoptamos una actitud más permisiva cuando leemos sobre la demolición del pabellón de servicios (al fondo) y la construcción de una nueva cocina en el lado norte del segundo patio hacia 1841. Es cierto que ese era el sector más precario, (donde la tapia no tendría casi cimientos), y con ello desaparecen los cuestionamientos; pero de la misma manera, si bien el resto del conjunto gozaba de más calidad, también era en cierta medida... precaria (al menos con más de cien años a cuestas) y, sin embargo, saber de su demolición pone en tela de juicio a sus responsables.

Con estas acciones y con esta parte de los argumentos se podría tender entonces a justificarlo, pero... el resto de los argumentos ¿qué dicen?, o en todo caso... ¿cómo responder otras preguntas de su accionar?

Luego de la compra por parte del estado y su reasignación a oficina de telégrafos, lo que se demolió fue precisamente el portal tan icónico con el que todos identificamos el edificio; justo el sector de mayor calidad constructiva (al estar cubierto con bovedilla). El resto del conjunto se conservó con trabajos de mantenimiento y dicho portal se reemplazó por lo que el estado entendía por “imagen institucional”, la fachada templaría neoclásica (ver figura 6).

Podríamos pensar que su demolición se debiera a que dicho sector tendría un deterioro irreversible, pero todas las fuentes coinciden en que el deterioro era general en todo el conjunto, ya que durante el periodo 1841-1870, la casa casi no tuvo mantenimiento (por estar a cargo de particulares con pocos recursos); o que en periodos anteriores (alquilada por el estado nacional) no se había hecho lo suficiente. La foto de Paganelli de 1869 (ver fig.4) evidencia el acentuado deterioro, pero el estado igualmente pudo conservar el conjunto, al menos 30 años más... excepto el emblemático portal.

Así, podemos citar palabras del propio Buschiazzo

“.... desgraciadamente, la pasada indiferencia de nuestras autoridades hacia tan vital problema ha sido la causa principal de la desaparición de muchísimos monumentos, algunos de alto valor; estos hechos vandálicos datan casi todos de los últimos treinta años, de modo que se habrían podido evitar si en ese entonces se hubiese puesto alguna atención y un poco de patriotismo bien entendido. El caso más lamentable es el de la casa donde se juró la independencia, en Tucumán......” (Buschiazzo, 1999:103)

Por ello es lógico entender que su demolición nada tuvo que ver con cuestiones materiales, sino por criterios en busca de una imagen institucional y que, si bien el edificio era importantísimo para el estado, no lo tomaron como museo, sino con un uso funcional. Entonces, ¿por qué el edificio era importantísimo para el estado sino fuera por razones patrimoniales? Y si era importante por estas razones ¿por qué no lo tomaron como museo o al menos intentaron mantener su imagen?

"La prensa local de la época hizo críticas a la obra, observando el descuido hacia la parte sobreviviente del edificio, aunque sin considerar la perdida de la tradicional fachada un problema relevante” (Páez de la Torre, 1986: s/e)

Es conveniente recordar que hacia 1870, aún seguía vigente el rechazo hacia la arquitectura del periodo colonial, y que el neoclasicismo seguía siendo la imagen adoptada por el estado, lo que podría sugerirnos que para entonces no consideraban al pórtico más importante que el resto del conjunto, o tal vez pensaran que solo el recinto del salón de sesiones fuese lo más importante, pero solo nos quedaron los hechos; no se registraron los motivos de esos hechos. 
De todos modos, a los ojos de nuestros días, este reemplazo puede considerarse un grave desacierto, pero también, a los ojos de nuestros días, tendría valor el pórtico neoclásico si hubiese subsistido (ver figura 6).

Lo paradójico aquí, es que el presunto rechazo, era hacia la imagen colonial que brindaba el pórtico original; pero a la vez la importancia de la casa era porque representaba la ruptura con ese pasado colonial. Y paradójico es también que el inicio de la valoración del portal, coincide con la decisión de su demolición, como si fuese necesaria su ausencia para recordar su valor, y las reproducciones de la única foto que se le tomo, la hubiesen hecho más presente que cuando existía físicamente.

La revalorización del portal se fue incrementando con el paso del tiempo, y se puede evidenciar destacando algunos sucesos:

- En 1872 fue la publicación de Arsenio de Granillo “La Provincia de Tucumán”, (figura 11) conteniendo las fotos de Paganelli, que comienzan a difundir la imagen del portal. (Ver figura 4).

- En 1890 ya se encuentran versiones de las fotos, coloreadas luego de la demolición.

- En 1893 (a 18 años de la desaparición del portal) la primera peregrinación estudiantil de la Unión Universitaria de Córdoba y Buenos Aires instalo sobre el salón una escenografía con un telón que reproducía el pórtico a escala natural, donde se lo rescata como símbolo de la independencia en la misma casa.

- A partir de allí surge el rito de la ofrenda de placas conmemorativas dentro del salón, donde muchas recreaban el desaparecido frente. Comienza una época con una visión romántica que reconocía a las ruinas como testimonio de un pasado valioso.

- En 1895 Museo Histórico Nacional adquiere un óleo de Genaro Pérez que representa de modo romántico la foto de Paganelli. Su difusión consolida la imagen del portal y sus ruinas (figura 10).

- En 1898 la Municipalidad de San Miguel de Tucumán adopta como escudo el símbolo que contenía el pórtico colonial, ya no en escorzo, sino frontal (figura 12).

- En 1900 el Pintor Pedro M. Medina presenta conmemorando el 9 de Julio, una representación del portal donde lo eleva a símbolo patrio, rodeado por la alegoría de la Republica, el Escudo nacional, la Bandera, los Escudos de las Provincias y los nombres de los congresales de 1816.

- En 1909 el tucumano Ricardo rojas publica “La Restauración Nacionalista” fomentando el interés hacia las raíces hispanoamericanas

- En 1916 Juan Kronfuss escribe "La Arquitectura Colonial en Argentina” (publicado en 1921) donde dibuja el portal reproduciendo todos los detalles y en geometral, pero ya no como parte de una casa sino con la categoría de ruina de un gran arco triunfal en un paisaje bucólico.

- En 1927 Ángel Guido recreo el pórtico de la casa Histórica en el frente de la casa que diseño para Ricardo Rojas en Buenos Aires- Hoy museo Ricardo Rojas (figura 13).

- En 1934 se replica el portal en la escuela General Belgrano, en Tucumán, construida por la Nación (figura 14)

- En 1938 Ángel Guido concluye el proyecto de "Sistematización Monumental de la Casa Histórica de Tucumán” con el proyecto de reconstrucción de la parte delantera.

- En 1940 se forma una subcomisión, entre la Comisión Nacional de Museos, Monumentos y Lugares Históricos, la Academia Nacional de la Historia, y la Dirección Nacional de Arquitectura, con Levene, Mario Buschiazzo, Martin Noel y Alejandro Figueroa destinada a la reconstrucción fiel de la Casa del Congreso de 1816 donde Buschiazzo busco toda la información posible para legitimar el proyecto y evitar caer en fantasías y falsedades.

- En 1943 se inaugura la mencionada reconstrucción. (figura 15). 


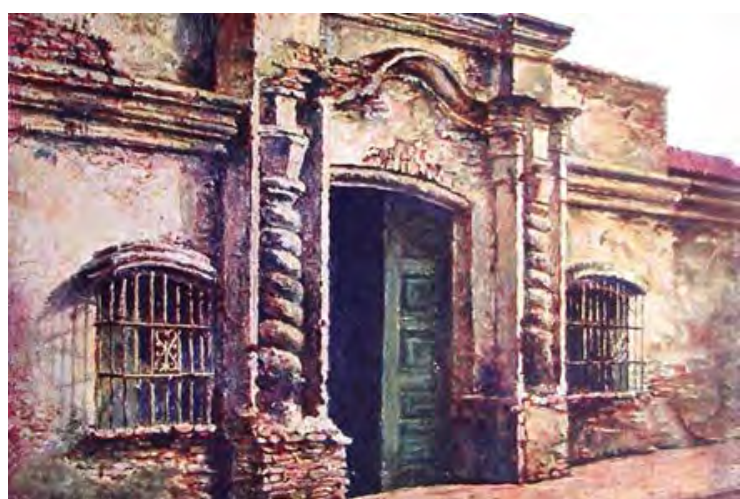

Figura 10. Oleo de Genaro Pérez (Fuente: diario La Gaceta $)^{4}$

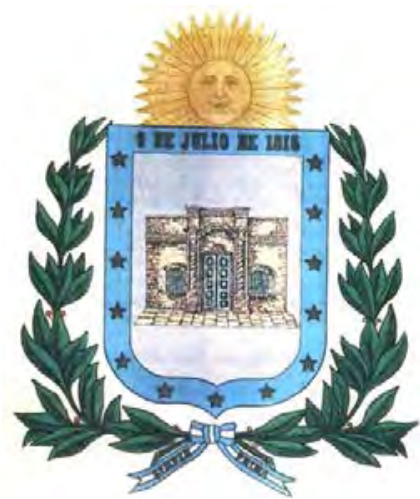

Figura 12. Escudo del Municipio de San Miguel de Tucumán (Fuente: sitio digital) ${ }^{6}$

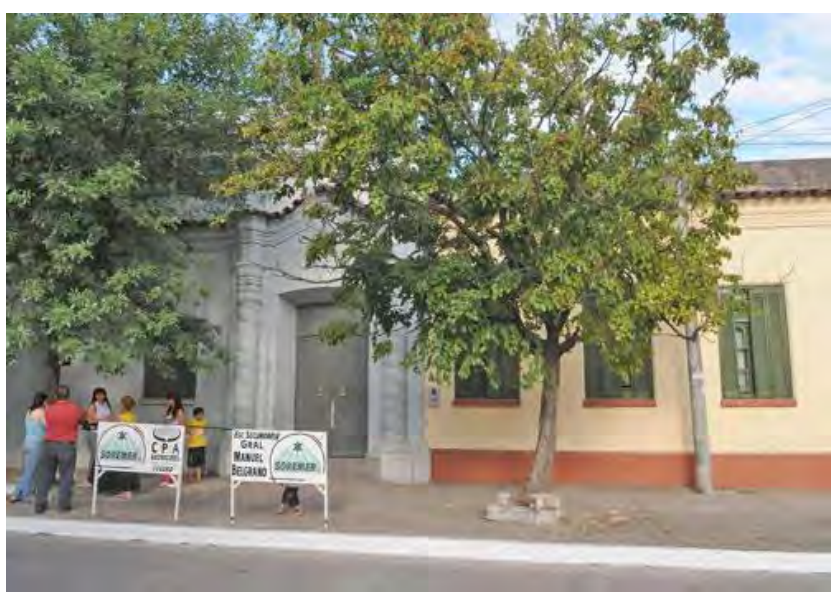

Figura 14. Escuela Gral. Belgrano en Tucumán (Fuente: diario La Gaceta) ${ }^{6}$



Figura 11. Publicación de Arsenio Granillo (Fuente: La Razón 1872) ${ }^{5}$

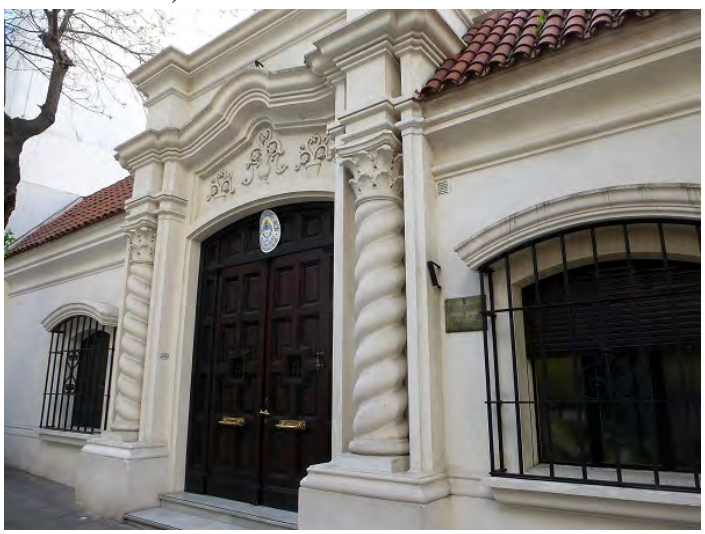

Figura 13. Casa de Ricardo Rojas (Fuente: Imagen propia)

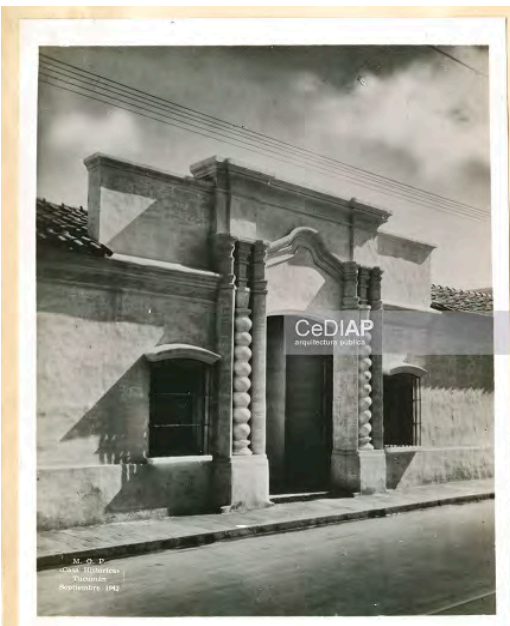

Figura 15. Foto posterior a su reconstrucción. (Fuente: CeDIAP)

Volviendo a los sucesos que afectaron a la casa, podemos continuar con la gran demolición de 1904, otra acción que nos llena de interrogantes, dado que los textos citados de Buschiazzo y de Marin- 
salda (ver bibliografía al pie) señalan que el edificio continuaba en estado ruinoso, pero no se encuentran registros el porqué. Si hacía más de treinta años que estaba bajo el “cuidado” del estado nacional, y justamente era el estado el que consideraba al inmueble con un alto valor emblemático. Es cierto que el tipo constructivo en tapia o adobe no era durable, y menos con el clima tucumano, pero era posible de conservarse con mantenimientos que implican casi una reconstrucción cotidiana.

Esto que nos lleva a otra pregunta ¿Qué tanto de original puede plantearse de un edificio en crónica reconstrucción? Ello se contestaría con recordar que existen muchos ejemplos de valor patrimonial con tecnologías de crónica reconstrucción; claro que, en esos casos mantener esa tecnología y su tradición constructiva (con los métodos originales), es lo que los carga de significado y su originalidad, puede mutar en tanto vaya mutando el método de mantenimiento, o la percepción de cuáles eran los "métodos originales".

Seguir profundizando en esto, seria alejarnos de nuestro caso, que fue afectado de la mencionada demolición y reemplazado por un envoltorio italianizante que existió solo para proteger a lo único que se conservó como a una valiosa perla, el recinto del salón de sesiones.

"Parece inconcebible que se haya permitido esa bárbara destrucción, para conservar tan solo el local donde se reunió la asamblea, protegida por un absurdo edificio de estilo francés, que lo recubre a manera de fanal" (Buschiazzo, 1999:103) (ver figura. 15)

Entonces, este salón, ¿era más importante que el resto del conjunto?, o al menos ¿más importante que la icónica fachada? O tal vez no, pero ya no la podían conservar a 30 años de su reemplazo.

En esta reflexión, poco habría que decir sobre el edificio italianizante como tal, ni sus cualidades artísticas, espaciales, etc. Sus breves 40 años de existencia se justificaron solo para conservar el pequeño recinto, que paso a ser valiosísimo, justamente por estar protegido por esta cascara y por ser lo único que se conservó del edificio original (figura 15).

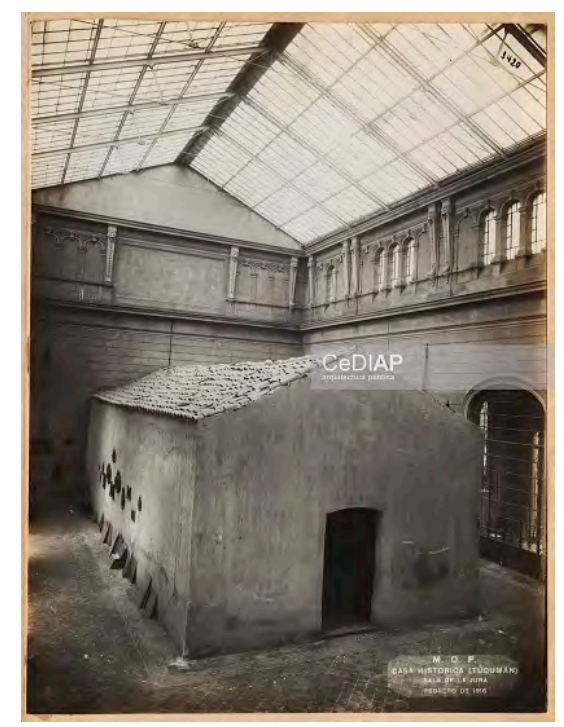

Figura 15. Salón de la jura dentro del pabellón ecléctico. (Fuente: CeDIAP) 
Igualmente, el recinto del salón de sesiones, también fue objeto de una restauración, que implico desarmar y reamar la mayor parte de su techumbre, y reintegrar sus muros, así como el reemplazo de muchas de sus partes, con la introducción de nuevas tecnologías que lo dotaron de mas estabilidad; por ejemplo, en el apoyo de la estructura del techo (figura 16). En cierta medida, el trabajo volcado sobre este sector, concuerda bastante con los criterios de intervención que se manejan hoy en día lo que nos deja con la pregunta: ¿Por qué no se pudo hacer lo mismo en el resto del edificio?

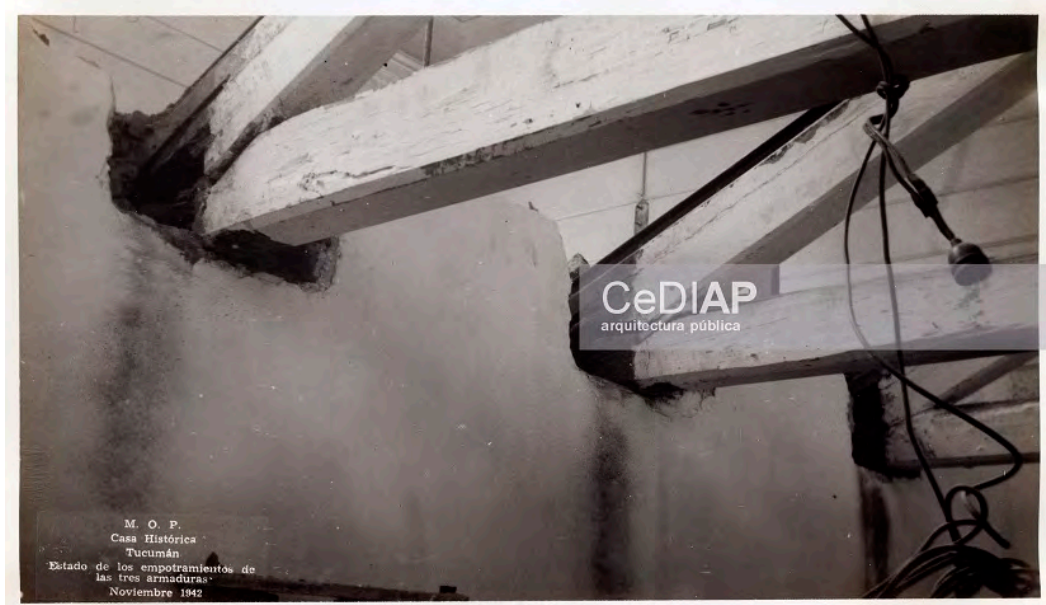

Figura 16. Salón de la jura dentro del pabellón ecléctico. (Fuente: CeDIAP)

Finalmente, la revalorización de la imagen nacional y el proceso de creciente importancia que fue ganando el añorado portal, concluyeron en la reconstrucción del caserón original, respetando con el mayor rigor que la investigación les permitió todos sus detalles, e inclusive usando los mismos materiales y técnicas que se usaron en el original (figura 17). Es muy sensible opinar sobre las acciones de profesionales tan célebres en nuestra historia, que inclusive son los primeros en trabajar sobre el patrimonio ${ }^{7}$ en nuestro país; pero a los criterios actuales, esta reconstrucción generó un objeto nuevo a imagen y semejanza del original y reemplazándolo en su lugar.

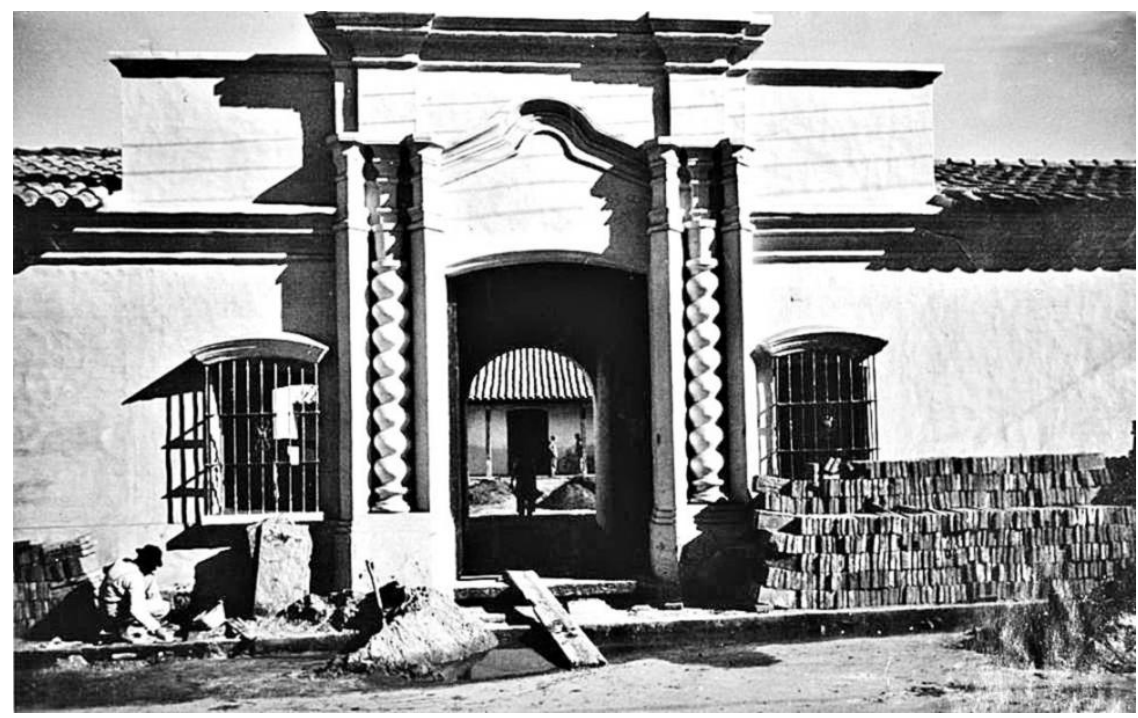

Figura 17. Durante la reconstrucción de 1943. (Fuente: Museo Casa Histórica de la Independencia) 
Teoría y Práctica de la Arqueología Histórica Latinoamericana | Año VIII. Vol. 8 | 2019

ISSN en línea: 2591-2801 | ISSN versión impresa: 2250-866X

Entonces el interrogante aquí es sobre sus cualidades patrimoniales, sabiendo que en términos físicos nada tiene del original (excepto el recinto del salón conservado) o que en todo caso sus cualidades patrimoniales no pasaron ni por la conservación ni por la restauración. Aquí se trata explícitamente del valor intangible de lo que representa como símbolo de lo que fue; ¿entonces, si ese valor es intangible ¿por qué se necesita el objeto tangible? ¿Por qué no basta con la divulgación de la imagen del original, o en todo caso con una exhibición museológica en el lugar que indique lo que allí hubo?

Para profundizar esta idea, podemos citar a Turner:

"Los símbolos patrios pueden ser denominados instrumentales, es decir poseen un significado independientemente del contexto en el que se encuentren, la bandera nacional representa a la identidad de ese país, así esta se encuentre en la inauguración de las olimpiadas, en un acto en conmemoración a los caídos de Malvinas o en la cancha de futbol. El significado de los objetos denominados patrimonio cultural si varía de acuerdo al contexto lo que permite relocalizarlos y resignificarlos en contextos espacio-temporales diferentes, de acuerdo al uso que se pretenda hacer de ellos.

Conviene, antes de seguir adelante, definir que entendemos por símbolos, ya que es un concepto polisémico cuyo significado varía de acuerdo al contexto teórico que lo rodea. Un "símbolo es una cosa de la que, por general consenso, se piensa que tipifica naturalmente, o representa, o recuerda algo, ya sea por la posesión de cualidades análogas, ya por asociación de hecho o de pensamiento” (Turner, 2002:21).

Siguiendo la definición propuesta, el sentido de un símbolo depende de un consenso, representa o recuerda a algo, ya sea por cualidades parecidas o conexiones ideales. "El poder de los símbolos reside en su capacidad de sintetizar una identidad para lo que pone en funciona-miento la lógica práctica, es decir principios generadores inconscientes que se pueden activar a través de múltiples analogías” (Bourdieu, 2007:211).

Por ello, no es lo mismo la reconstrucción de la Casa Histórica tal como fuese la original y en su sitio, que por ejemplo su reproducción en el ingreso del museo Ricardo Rojas (ver figura 13) En este sentido el patrimonio cultural presta un servicio al dispositivo ${ }^{8}$ generador de identidades que los símbolos patrios no pueden realizar.

Pero volviendo a los criterios de intervención mencionados en las cartas internacionales, estas no presentan a la “reconstrucción” como una intervención patrimonial. De hecho, no la mencionan. Basan a la gestión patrimonial principalmente en la conservación, y en un extremo en la restauración. Así, por ejemplo, la Carta de Venecia en el Art.9 expresa: "La restauración es una operación que debe tener un carácter excepcional. Tiene como fin conservar y revelar los valores estéticos e históricos de un monumento y se fundamenta en el respeto hacia los elementos antiguos y las partes auténticas. Se detiene en el momento en que comienza la hipótesis; más allá todo complemento reconocido como indispensable, se destacará de la composición arquitectónica y llevará el sello de nuestro tiempo ${ }^{9}$. De hecho, en 1978, la misma Carta Internacional para la Conservación y Restauración de Sitios y Monumentos retomaba este asunto diciendo: "La restauración se funda en el respeto hacia la sustancia original o antigua del monumento o sitio y sobre los documentos auténticos que le conciernen ${ }^{10}$. Esto marca la necesidad de que al restaurar edificios y otros bienes culturales tangibles se protejan, tanto el soporte material, como la autenticidad, principios que fueron reafirmados posteriormente por otros documentos internacionales. Entre ellos se cuentan las resoluciones adoptadas en el Coloquio sobre "La Conservación, Preservación y Valorización de Mo- 
numentos y Sitios en función del desarrollo del turismo cultural”, que tuvo lugar en Oxford, en julio de $1969^{11}$ la Carta Internacional para la Conservación y Restauración de Sitios y Monumentos ${ }^{12}$; el Documento de Nara ${ }^{13}$ y el Documento Regional del Cono Sur sobre Autenticidad, conocido también como la Carta de Brasilia ${ }^{14}$.

La necesidad de la construcción del nuevo caserón imitando las técnicas originales y de un modo tan logrado, podría llegar a considerarse un "falso histórico". Queda claro que no lo es ya que, todos los procesos que padeció están documentados y divulgados con extensa bibliografía, pero a excepción de una leve información exhibida en el lugar, se podría asegurar que la mayoría de las publicaciones que tratan su reconstrucción, pertenecen al ámbito académico, y que quienes no están involucrados con la temática ignoran que no se trata del original; y nuevamente cabría preguntarnos ¿qué tan divulgada esta esta obra de reemplazo?

¿Y por no ser el original, deja de ser autentico? Recordemos que, al respecto, la Declaración de San Antonio expresa: "La autenticidad de nuestro patrimonio cultural está directamente relacionada con nuestra identidad cultural.” (ICOMOS; Declaración de San Antonio, op. cit., Art. 1.)

Por aquello de lo que se destruye no tiene vuelta atrás, aquí si la tuvo; y también... así como surgen todos estos interrogantes: ¿qué tan prudente fue la demolición del pabellón italianizante construido en 1904? En general no se menciona esta demolición, y los profesionales en patrimonio se enfocan en la obra neocolonial.

Cabe recordar que el propio Buschiazzo, en su artículo sobre la destrucción de los monumentos, desarrolla en extenso la idea de "restauración”, por ejemplo, para el cabildo de Buenos Aires, pero como el artículo es previo a la reconstrucción que realizo en nuestro caso, no citó ningún ejemplo que lo lleve a exponer su posición sobre estos procedimientos.

Aquí, ni nos detuvimos en describir el pabellón italianizante, pero también forma parte de nuestra historia. Es muy pertinente en esta ocasión citar el artículo 11 de la Carta de Venecia que habla de la Patina con "las valiosas aportaciones de todas las épocas" que sugiere respetar, ya "que la unidad de estilo no es un fin a conseguir en una obra de restauración”. Tal vez hubiese sido más noble conservar dicho pabellón, y emplearlo no solo para conservar el salón histórico, sino para exhibir todos los documentos que recuerden como fue el original anterior a él.

Pero, de todos modos, la situación actual presenta la existencia de la réplica de 1943, y luego de preguntarnos que debió haberse hecho, debemos preguntarnos qué debe hacerse ahora, ya que aparte de todo su significado simbólico, el objeto fisco también forma parte de "la pátina del tiempo" y por eso lo que cabe es una única respuesta. Conservarlo sin dudas.

\section{Notas}

${ }^{1}$ Archivo del Centro de Documentación e Investigación de la Arquitectura Pública (CeDIAP) del Ministerio de Economía y Finanzas Públicas.

${ }^{2}$ El revoque de barro blanqueado sobre tapial se verifico en los cateos de 1993 en sectores de la sala

${ }^{3}$ Buzchiazzo, M. (1999) La destrucción de nuestros monumentos. Anales del Instituto de Arte Americano (31 y 32): 95-106.

${ }^{4}$ https://www.lagaceta.com.ar/nota/737865/opinion/aquel-famoso-congreso.html

${ }^{5}$ Granillo, A. (1872) Provincia de Tucumán - Serie de Artículos descriptivos y noticiosos. Tucumán, Argentina: La Razón.

${ }^{6}$ https://es.wikipedia.org/wiki/Escudo_de_San_Miguel_de_Tucum\%C3\%A1n 
${ }^{7}$ Según las definiciones contenidas en el diccionario de la RAE, podemos decir que patrimonio es, en términos generales, el conjunto de los bienes heredados o adquiridos, asumidos como propios y susceptibles de estimación económica. 9 http://lema.rae.es/drae/?val=patrimonio. En base a esto, Patrimonio Cultural se define como el conjunto de bienes heredados de nuestros antecesores que identifican a un determinado grupo social, les resultan propios y característicos, y en tanto ello, de algún modo diferenciadores de otros grupos, dándoles a sus integrantes cierto sentido de pertenencia. El contenido de aquello que se entiende como patrimonio es resultado de la creatividad artística, tecnológica, cultural, etc. y se transmite de una generación a la otra a lo largo de tiempo, a la vez que se re significa. Carta ICOMOS (2003) Principios para el Análisis. Conservación y Restauración de las Estructuras del Patrimonio Arquitectónico. Ratificada por la $14^{\circ}$ Asamblea General del ICOMOS, Zimbabwe, Art. 1.2

${ }^{8}$ Utilizamos el concepto de dispositivo en el sentido elaborado por Foucault. "se caracteriza por desplegar dispositivos (...) que son un conjunto decididamente heterogéneo, que comprende discursos, instituciones, instalaciones arquitectónicas, decisiones reglamentarias, leyes, medidas administrativas...” (Foucault, M. 1991: 128)

${ }^{9}$ ICOMOS; Carta Internacional sobre la Conservación y la Restauración de Monumentos y Sitios (Carta de Venecia): II Congreso Internacional de Arquitectos y Técnicos de Monumentos Históricos, Venecia, 1964. http://www.icomos.org/charters/venice_sp.pdf

${ }^{10}$ ICOMOS; Carta de Burra. Burra (Australia), agosto 1979. Actualizada en febrero de 1981, abril de 1988 y noviembre de 1999. Art. 3.1

${ }^{11}$ Díaz-Berrio, F. (1974) Terminología general en materia de conservación del patrimonio cultural prehispánico. México. XLI Congreso Internacional de Americanistas, p. 4

${ }^{12}$ Op. Cit. 12

${ }^{13}$ Op. Cit. 12

${ }^{14}$ Op. Cit. 12

\section{Referencias bibliográficas}

KRONFUSS, J. (1934) Arquitectura Colonial en la Argentina. Córdoba: Argentina: Biffignandi.

BUSCHIAZZO, M. J. (1940). La destrucción de nuestros monumentos históricos. Revista de Arquitectura, (230).

MARINSALDA, J.C. (1999). La Casa Histórica de la Independencia de 1816 y la reconstrucción de Mario J. Buschiazzo. Anales del Instituto de Arte Americano (31 y 32) 155-184

BOURDIEU, P. (2007). El sentido práctico. España. Taurus Humanidades.

FOUCAULT, M. (1991). Saber y verdad. España. La piqueta.

TURNER, V. (2002). Selva de Símbolos. España. Siglo XXI.

DÍAZ-BERRIO, F. (1974). Terminología general en materia de conservación del patrimonio cultural prehispánico. México. XLI Congreso Internacional de Americanistas

CARTA ICOMOS (2003). Principios para el Análisis. Conservación y Restauración de las Estructuras del Patrimonio Arquitectónico. Ratificada por la $14^{\circ}$ Asamblea General del ICOMOS, Zimbabwe, Art. 1.2 
HISTORIA DE LA CASA (2018). Museo Casa Histórica de la Independencia. https://casadelaindependencia.cultura.gob.ar/.

PÁEZ DE LA TORRE (H), C. (1986). La Casa Histórica a través de los años. San Miguel de Tucumán, Argentina: Archivo C.N.M.M.Y. y L.H. Legajos Casa Histórica de la Independencia, Nro 1 al 8. Archivo fotográfico (sin clasificar).

ARCHIVO DEL CENTRO DE DOCUMENTACIÓN E INVESTIGACIÓN DE LA ARQUITECTURA PÚBLICA (CeDIAP) del Ministerio de Economía y Finanzas Públicas.

Recibido: 4 de julio de 2019

Aceptado: 10 de septiembre de 2019 